\title{
Mov10, an APOBEC3G-interacting RNA-binding protein, inhibits HIV-1 infection
}

\author{
Shetal Arjan", Chad Swanson, Nathan Sherer, Michael Malim \\ From Frontiers of Retrovirology 2011 \\ Amsterdam, The Netherlands. 3-5 October 2011
}

\begin{abstract}
Background
Identification of cellular factors that negatively or positively regulate HIV-1 replication is essential to understand HIV-1 pathogenesis for development of novel antiviral treatments. The human cytidine deaminase APOBEC3G (A3G) is an intrinsic antiviral factor important for the host-mediated defence against HIV-1. We and others recently identified a panel of A3G-interacting RNA-binding proteins (RBPs) that focused our search for novel HIV-1 cofactors and restriction factors onto these proteins. Mov10, a superfamily-1 putative RNA helicase, interacts with A3G in an RNA-dependent manner. Mov10 associates with the RNA-induced silencing complex pathway and localises to mRNA processing bodies (PBs). Furthermore, orthologs of Mov10 in Arabidopsis thaliana and Drosophila melanogaster are necessary for antiviral small RNA-mediated silencing. Thus, we investigated whether Mov10 restricts HIV-1 replication.
\end{abstract}

\section{Materials and methods}

The effect of Mov10 on HIV-1 virus production and ínfectivity was determined by ectopic expression and silencing of Mov10 in virus-producing HeLa and 293T cell lines. Cells were transfected with a plasmid encoding the full-length HIV-1 $1_{\mathrm{NL} 4-3}$ provirus or infected with VSV-G pseudotyped HIV-1 $1_{\text {NL4-3 }}$ virus. Virion production was measured by $24^{\mathrm{Gag}}$ ELISA and infectivity was determined by infecting a TZM reporter cell line with virus normalised by $\mathrm{p}^{\mathrm{G}}{ }^{\mathrm{Gag}}$ concentration.

\section{Results}

The ectopic expression of Mov10 substantially decreased HIV-1 virion infectivity, whilst moderately decreasing

Department of Infectious Diseases, King's College London, London SE1 9RT, UK virus production. Mov10 induced a significant reduction in the accumulation of minus strand strong stop DNA in the target cell, which likely accounted for the considerable loss in virion infectivity. Preliminary results showed a small decrease in HIV-1 genome incorporation into budding virions that may have contributed to the defect at strong stop. Mov10 comprises a 495 residue amino-terminal domain with no known protein motifs, and a 508 residue carboxy-terminal putative RNA helicase domain. To begin to identify important attributes in Mov10 we mutated known helicase motifs in the full-length protein; overexpression of these mutants determined that the carboxy-terminal putative RNA helicase domain of Mov10 is necessary for its antiviral activity. Furthermore, RNAi-mediated silencing of endogenous Mov10 moderately enhanced HIV-1 virion production with no discernable effect on virion infectivity. We also tested A3G antiviral activity in the context of Mov10 silencing, which was unaffected.

\section{Conclusions}

Our findings indicate a potential antiviral role for Mov10 in the HIV-1 life cycle, independent of A3G antiviral activity. The effect of Mov10 silencing on HIV1 was modest, however, this is currently being addressed in primary $\mathrm{CD} 4^{+} \mathrm{T}$ cells and macrophages, the natural targets of HIV-1 infection. Furthermore, we are investigating whether Mov10 is upregulated by type I interferon and identifying molecular mechanism/s by which Mov10 is able to inhibit HIV-1 infection.

Published: 3 October 2011

doi:10.1186/1742-4690-8-S2-P2

Cite this article as: Arjan et al:: Mov10, an APOBEC3G-interacting RNAbinding protein, inhibits HIV-1 infection. Retrovirology 2011 8(Suppl 2):P2.

\section{Biomed Central}

(c) 2011 Arjan et al; licensee BioMed Central Ltd. This is an open access article distributed under the terms of the Creative Commons Attribution License (http://creativecommons.org/licenses/by/2.0), which permits unrestricted use, distribution, and reproduction in any medium, provided the original work is properly cited. 